\title{
A NEW INSTRUMENT FOR DARK ADAPTATION TESTS*
}

\author{
BY \\ W. $\mathrm{KOCH}$ \\ DEPARTMENT OF HYGIENE, THE HEBREW \\ UNIVERSITY, JERUSALEM
}

SEVERAL instruments are known for testing dark adaptation, Nagel $^{1}$, Feldmann ${ }^{2}$, Jeans, Blanchard and Zentmire ${ }^{3}$ and Hecht and Shlaer ${ }^{4}$, each described a type of apparatus for determining the light threshold in dark adaptation (minimum percepible).

The most reliable of these instruments is that of Hecht and Shlaer. However, when Hunt and Palmer ${ }^{5}$ tested simultaneously a series of people with three different instruments of the same type they did not obtain identical findings. Only after some modifications they succeeded in making these instruments give concordant results.

It is intended to describe below a simpler instrument which is relatively cheap and easy to construct and at the same time appears reasonably reliable.

\section{Description of the Instrument}

The instrument consists of an oblong box (1), one end of which (2) fits the eyes and nose, similar to a stereo-viewer, but without lenses. A piece of metal (3) may be shifted over one hole so that only one eye may be tested.

The box is about $35 \mathrm{~cm}$. long and its end is covered by a clear. glass plate (4). At the right and left ends of the plate, $5 \mathrm{~cm}$. from the centre, is a number in Indian Ink (5 r) and (5 1) (in the drawing represented by filled half circles), which serves as a fixation point, for the right and the left eye respectively.

The next part of the instrument is a camera-like box (6) which is hinged (Photos) and can thus be removed from the path of the rays. The end of this box facing the glass plate (4) is closed with a black cardboard (7), with a central hole $14 \mathrm{~mm}$. in diameter. To the right and left from the centre of the hole is a fluorescent figure $(8 \mathrm{r}, 8 \mathrm{l})$ (empty half circles), which adjoin the Indian Ink figures on the glass plate of the viewing box when that and the camera-like box are brought into line. (Drawings $\mathrm{I}^{\prime}$ and III.) These fluorescent figures serve as fixation points in the dark. Into the camera-like box plates are inserted. A blue glass plate (9), a series of ground glass and opalescent glass plates (10); these

* Received for publication, September 20, 1944.

* See photos and drawings. 


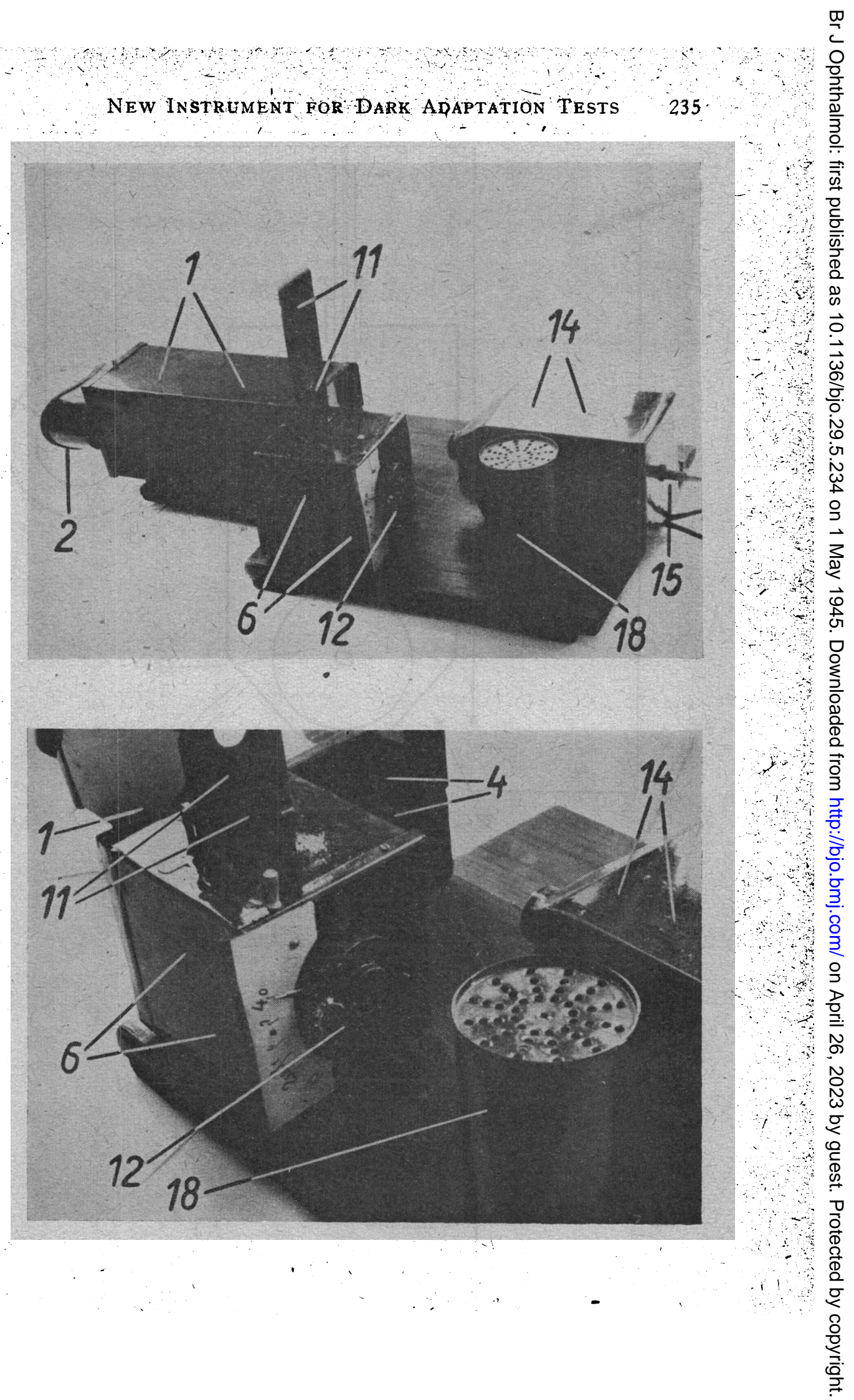




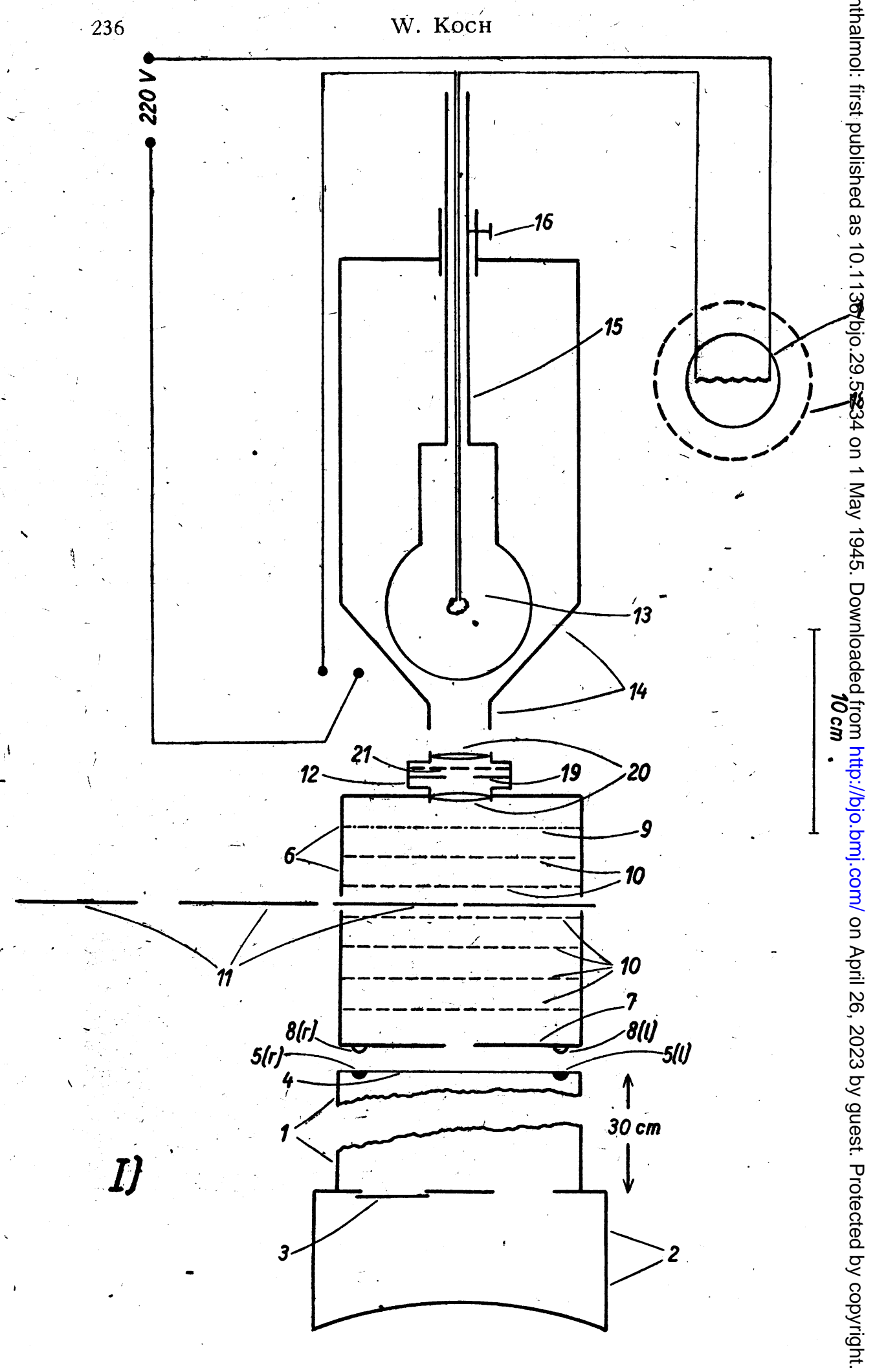


plates serve to diffuse the light and to reduce its intensity: A strip of metal (11) with three holes of various sizes, having a relationship of $1: 10: 100$ is inserted like a lantern slide into the box. These holes reduce the intensity of light in predetermined proportionate amounts. The upper edge of the strip bears three dentations, while the box carries their counterpart; a flat spring and a tooth, similar to the arrangement which keeps the objective lenses in the nose piece of a microscope in position. Into the wall opposite the cardboard (7), an ordinary Compur shutter (12) is inserted.

The third part is the frosted lamp (13) (25 watts, 110 volts), inserted into a box (14) by a rod (15) bearing a $\mathrm{cm}$. scale. By moving the rod which can be fixed by a screw (16), the lamp can be brought nearer or further from the shutter. This lamp is connected in series to a regulator lamp (17) (iron resistance lamp Philips $\mathrm{C}_{1}$ ) which assures a constant voltage (101 volts, plus/minus 1 when employing ca. $220 \mathrm{v}$.). The resistance lamp is protected by a metal cover (18):

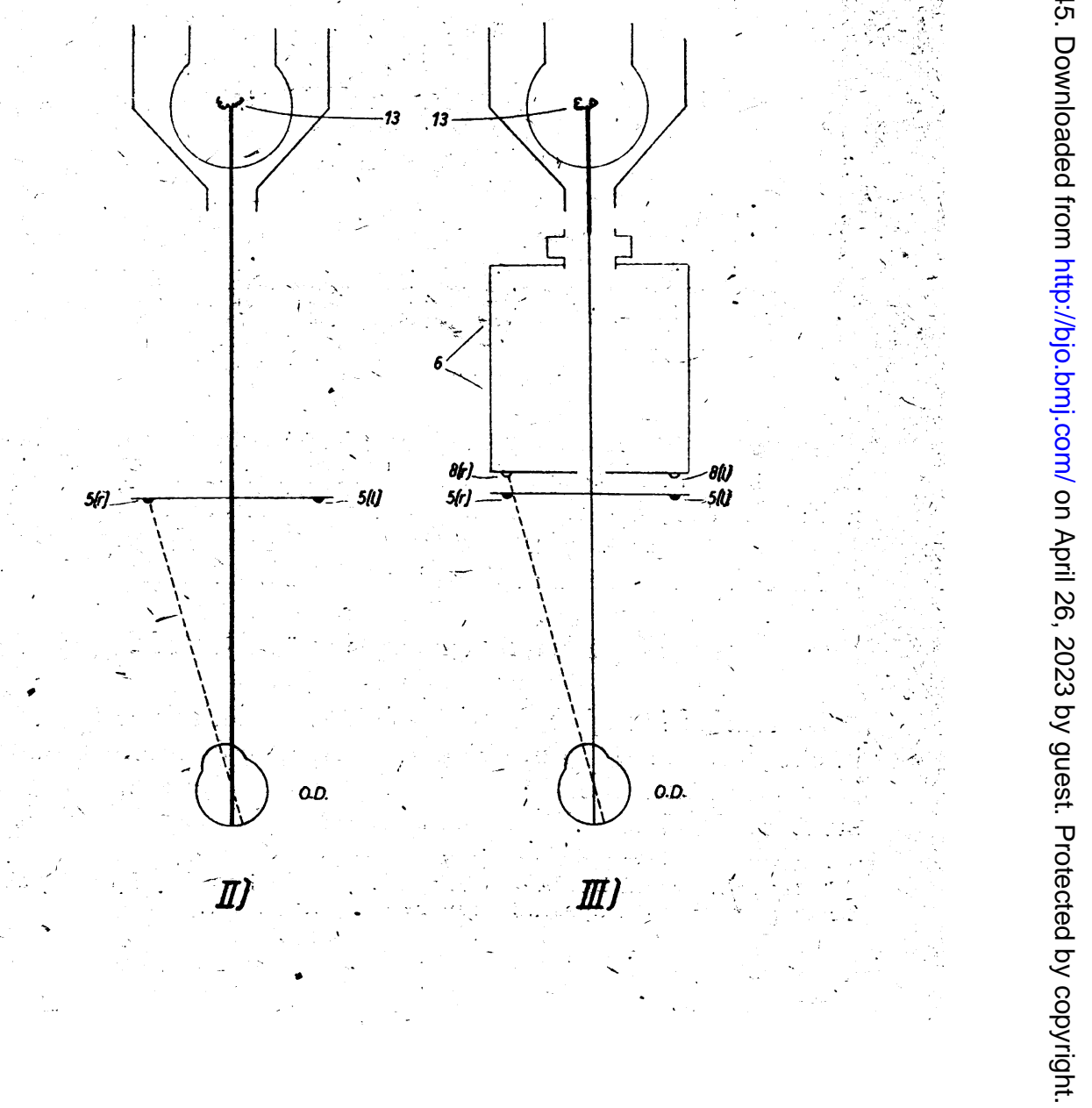


- Calibration of the Instrument-Since an optical check of the instrument is not easily available, the base of our scale is fixed by the mean light threshold of young healthy persons after sitting in the dark for 30 min.* According to Hunt and Palmer (1.c.) the mean of this threshold is $2.5 \mathrm{log}$ units of millimicrolamberts.

A diaphragm is considered to be one-of the most reliable means of reducing the intensity of light (Sewig6). . An ordinary camera

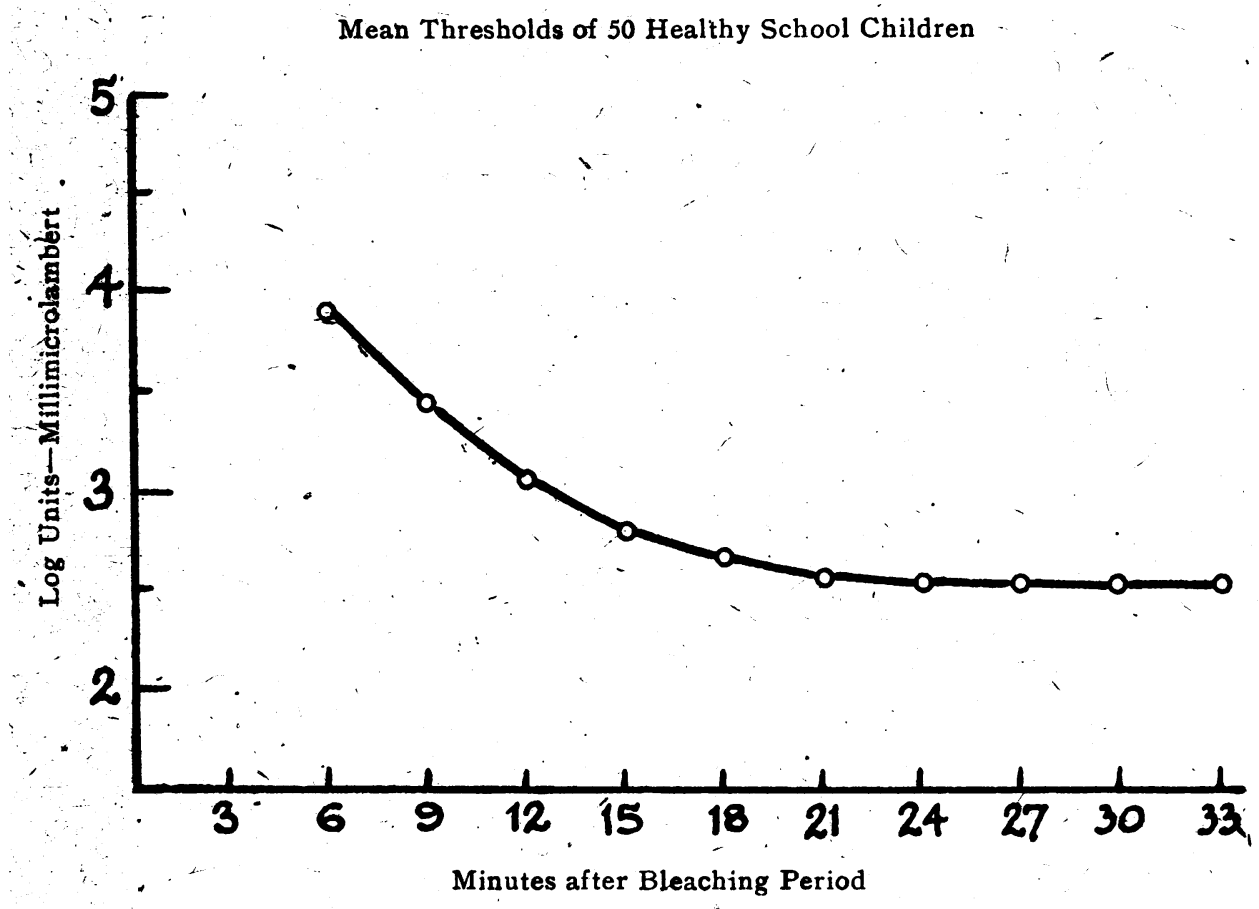

diaphragm (19) bears a set of figures so arranged that on stopping down each subsequent figure reduces the intensity of light by half. In the objective lens (20) the figures of the stop are :

$$
f: 32 \text { f }: 23 \text { f: }: 16 \text { f }: 11 \text { f }: 8 \quad f: 6 \cdot 3
$$

Therefore the ratios of light intensities are :

$$
1: 2: 4: 8: 16: 32
$$

or in logarithmic values, since the log of $2=0.3$

$$
\begin{array}{lllllll}
0 & 0.3 & 0.6 & 0.9 & 1.2 & 1.5
\end{array}
$$

-Determination of the Basic Point.-A healthy young person sits . in the dark for $30 \mathrm{~min}$. The smallest hole of the metal strip (11) is brought into the axis of the apparatus, the diaphragm (19) is stopped down to $f: 32$, and the shutter (21) set as to deliver half

* Meanwhile word has been received from the National Physical Laboratory. Teddington, that the apparatus can be calibrated there. 
a-second. Then he is asked to look with his right eye at the fixation point (fluorescent figure $8 \mathrm{r}$ ). The shutter is then wound and released. Three pošsibilities exist :

(1) The person may be unable to see anything.

(2) The person may see a bright flash.

(3) The person may see a faint flash, just visible, without form. In case (1); one or more ground glasses are removed from the box, until the response 3 is obtained. In case (2) ground glasses are added to the box till response 3 is achieved.' The final adjustment can be performed by moving the rod (15) of the lamp. A reading of the position of the lamp rod (15) is now taken and the rod fixed in position; taking other persons the test is repeated.

This-opening of the diaphragm ( $f: 32)$ thus established gives us the normar $30 \mathrm{~min}$. threshold value and is known to correspond to a light threshold of about $2.5 \mathrm{tog}$ units of millimicrolamberts (5). Since $f: 32$ has been fixed to correspond to $2 \cdot 5$, the subsequent figure of the stop, $f: 23$, corresponds to 2.8 (see last line of our chart), $f: 16$ to $3 \cdot 1 ; \dot{f}: 11$ to $3 \cdot 4 ; f: 8$ to $3 \cdot 7 ; f: 6 \cdot 3$ to 4.0 , These figures are entered on a cardboard attached to the face of our shutter (see photos); this cardboard is the scale of our instrument. Since our scale covers a range from 4:0-2.5 log units it can be seen from the graph based on the findings in normal children that the dark adaptation can be tested from the 10th minute. onwards.

In order to test the whole range of dark adaptation following. exposure to light an extension of the scale above 4.0 is necessary. This extension can be easily obtained by bringing the middlesized hole or the biggest hole of the metal strip into position. Taking the middle-sized hole the increase in light is tenfold, taking the biggest hole the increase in light is hundredfold. Since the logarithm of 10 is 1 , and the logarithm of $100=2$, the range is 25-4.0 log units-with the first hole, 3:5-5:0 when using the second hole, and 4.5-6.0 when using the third hole.

The arrangement can be checked as follows: Small pieces of photographic film, about $3 \times 3 \mathrm{~cm}$. in size are cut. The apparatus is put in the dark and the pieces of film are pasted on to the hole in the cardboard (7). Within the range of the film, the product of exposure time $\mathbf{x}$ intensity of light is constant. Hence the film should show a black circle of the same shade, when the smallest hole of the strip is exposed for a given time, as when the next hole is used with an exposure time of one-tenth. Slight differences may arise from the fact that a small hole is more difficult to centre than a bigger one; it has been found, however, that there is no real difficulty when a $2 \mathrm{~mm}$. hole is used, $2 \mathrm{~mm} ., 6 \cdot 3 \mathrm{~mm}$. and $20 \mathrm{~mm}$. being a rather useful set of diameters; smaller sizes are not feasible. 


\section{Sources of Error}

(1) Shutter.-As a time-factor is involved in our test it is necessary to make sure that a good quality of shutter is used. A Compur shutter meets this requirement. Slight deviations in the time delivered have no serious effect on the results, since a flash which cannot be seen at an exposure time of half a second will not be seen at a longer exposure time either.

(2) Varying Intensity of Light.- (a) Fluctuations in Voltage : Small changes in voltage induce marked changes in the output and composition of light. This difficulty is overcome by inserting an iron resistance lamp. The effect of changes in current is further reduced by keeping the lamp on a somewhat lower voltage than stated on the bulb (in our apparatus $101 \mathrm{v}$. instead of $110 \mathrm{v}$.), as a variation of say $100 . \mathrm{v}$. plus/minus 5 does not alter the light output to the same extent as $110 \mathrm{v}$. plus/minus 5 .

(b) B.y using a seasoned bulb, kept on a lower voltage as mentioned above, no marked dectrease'with age should occur.

\section{Performance of Test}

(I) The apparatus is put up in a dark-room.

(2) For the bleaching period the camera-like box is tilted to the side and thus removed from the path of rays.

(3) If the right eye is to be tested, the metal shutter, contained in the eye-piece is shifted as to cover the left eye.

(4) The person looks with his right eye at the Indian Ink figure on the glass-plate $(5 \mathrm{~F})$ for three minutes; an area of the retina corresponding to the size of the opening of the lamp-box is thus bleached.

(5) The eyes are covered so as to prevent further effect of light.

(6) The fluorescent figure $(8 r)$ is exposed to the light of an ordinary lamp or torch to intensify the fluorescence of the fixation poińt.

(7) The camera-like box is now tilted back into normal position, the $20 \mathrm{~mm}$. hole of the metal strip centered, the diaphragm of the shutter completely opened (to number 4.0 ), and the shutter set to deliver half a second.

(8) The bandage over the eyes of the subject is now removed and the patient asked to fix his eye on the fluorescent figure $(8 \mathrm{r})$.

- Now the shutter is wound and released. Between 3-5 minutes after bleaching the normal person should be able to see the light flash when looking at the fluorescent fixation point. If he does; the diaphragm is stopped down to meet the progressive increase in dark-adaptation.

(9) When a small diaphragm opening is reached the middlesized hole is centered by shifting the metal-strip (11). Then the 
diaphragm opening is increased ten-times (or bne log unit), and the test continued by reducing the diaphragm opening.

(10) Again, when a small opening of the diaphragm is reached which emits a visible flash, the smallest hole of the metal strip is centered and the process repeated.

(11) The number on the cardboard gives the direct readings of the threshold print when the smallest hole is used. When the largest hole of the strip is used, two should be added to each figure, and when the middle-sized hole is used, one should be added.

\section{Practical Hints}

(1) Arranging the tests.-The most stable reading among all thresholds is the final threshold. While according to, Hunt and Palmer the variability in repeated tests on the same person is about $0.56 \log$ units after ten minutes, the differences occurring after $30-33$ minutes are only plus/minus $0 \cdot 1 \log$ units. Though a poor dark adaptation can be assumed when it takes a longer time to arrive at $e . g ., 4.0 \mathrm{log}$ units, compared with normal cases, there is, on the other hand, the possibility that complete'bleaching of the purple was prevented by closing the eye. In the latter case a person. with a poor dark adaptation will quickly be able to give low readings. After about thirty minutes, however, the findings are practically independent of the intensities of light employed in preadaptation, and this increases the value of the results.

(2) Testing some people simultaneously.-Since the final threshold reached after about 30-40 minutes is very reliable and highly specific, the following technique was employed with good - results in a survey of children : Each child was bleached for three minutes, then the eyes, covered with a bandage or a handkerchief and the next one taken for bleaching and so on. After thirty minutes or more the final threshold of the first child was determined. Children yielding high thresholds were submitted to repeated tests to exclude the possibility of lack of collaboration. If high readings were again found, a complete curve was plotted. (s.b.).

(3) The answers.-According to Hunt and Palmer (1.c.) there is a difference between " Image just perceptible, Form and colour absent" and "definitely perceptible" of about 0.2 log units; another $0 \cdot 2$ between "definitely perceptible", and "form present "; 0.2 more between this perception and that defined as " bright image with form." According to our experience the patient will yield slightly better readings when approaching the final threshold from the visible side. Therefore, to give the person 
an orientation of what is going on, it will be found, best to deliver a bright flash: of say $5.0 \mathrm{log}$ units; he will then know where to locate the flash. Then we shift our metal-strip to the smallest hole, close down the diaphragm and ask the person to be tested to respond to the faintest visible flash. Now the final threshold is approached from the underthreshold side. Eight to ten flashes of different intensity are delivered and the figure of least intensity taken as the final threshold. This figure should be confirmed by two or three changes from " yes " to " no."

(4) Fatigue.- It is of no use to ask the person too frequently, since the answers will become less clear. It is by far better to give him three minutes to rest with closed eyes in the dark, and then to ask once more.

(5) Size of pupil.--Provided the pupil is not rigid the result will not be appreciably affected. According to Reeves $^{8}$ the pupil reaches its full size after five minutes in the dark.

(6) Plotting the complete' curve.-In. the beginning of an adaptation test it will be seen that recovery proceeds very quickly. Therefore it is more convenient, not to determine the light threshofd at intervals of three minutes as it is usually done but to release the shutter repeatedly and to mark the time when a given threshold (5.0, etc.) is reached. The dark adaptation curve itself is plotted with time as abscissa and log units of millimicrolamberts as ordinata.

\section{Findings}

The calibration of our apparatus is based on the assumption that the average final threshold of healthy children is $2.5 \mathrm{log}$ units of millimicrolamberts (graph 1).

After the calibration, a group of 352 normal children, aged 6-14 years were tested and these also yielded a mean final threshold of 25 , the standard deviation being 0.161 . - Data on standard deviations for children are not available, but our figure corresponds reasonably, well with those of adults reported by Hunt and Hayden ${ }^{10}$. These authors found among subjects of less than 40 'years a standard deviation of 0.250 (Mean : 2.698), and among subjects 40 years and older a standard deviation of 0.328 (Mean : 3.010). Michaelson ${ }^{11}$ - working with our apparatus gives in a recent paper a mean of 3.0 and a standard deviation. of. 0.33 as normal for adults.

Taking the mean plus twice the standard deviation as the probable limit of normality, a final threshold over 2.82 in our apparatus may be considered as suspicious, while a threshold over 2.98 (mean plus three times standard deviation) is definitely abnormal for children. For normal limits in adults see Michaelson ${ }^{11}$. 
Acknowledgment.- I wish to thank Prof. I. J. Kligler, Department of Hygiene, Hebrew University; under whose supervision the work has been, carried out, for his constant help and advice; sincerest thanks are also due to Prof. S. Hecht, Columbia University, for his helpful criticišm.

\section{REFERENCES}

1. Nagel, W. A.-Zwei Ápparate für die Augenärztliche Funktionsprüfung. Zeitschr. f. Augenheilk., Vol. XVII, 1907.

2. FeldmanN, J. B.-Practice of dark adaptation. Arch. Ophthal., Vol. XIX, 1938.

3. Jeans, P. C., Blanchard, E. and Zentmire, Z.-Dark adaptation and vitamin A. Jl. Amer. Med. Assoc., Vol. CVIII, p. 451, 1937.

4. HECHT, S. and SHLAER, S.-An adaptometer for measuring human dark adaptation. Jl. Optical Soc., Vol. XXVIII, 1938.

5. Hunt, E. P. and Palmer, C. E.-Medical evaluation of nutritional status Milbank Memorial Fund Quarterly, Vol. XVIII, No. 4, 1940.

6. SEWIG, R.-Objektive Photometrie. Julius Springer, Berlin, 1935.

7. Graham, C. H. and Margaria, R.-Area and the intensity-time relation in the peripheral retina: Amer. $J l$. Physiol., Vol. CXIII, 1935.

8. ReEves, P.-Rate of pupillary dilatation and contraction. Psychol. Rev.., Vol: XXV, No. 4, 1918.

9. PALMER, C. E.-Dark adaptation characteristics of private school children measured with the adaptometer. Amer.Jl. Public Health, Vol. XXXI, 1941.

10. HUNT, E. P. ard HAYDEN, K. M. $\rightarrow$ Medical evaluation of nutritional status. Milbank Memorial Fund Quarterly, Vol. XX, No. 2, 1942.

11. Michaelson, I. C.-Defective night vision among soldiers: dark adaptation results and their use in diagnosis. Brit. Jl. Ophthal., Vol. XXVIII, No. 3, 1944 .

\section{EXPLANATION OF FIGURES}

Drawing I gives the details of the apparatus. Drawing II, corresponding to the photos shows the bleaching of the retina, Drawing III, like Drawing I, the determination of the threshold.

1. Oblong viewing box.

2. Attachment to fit the eyes and nose.

3. Metal piece to cover one eye.

4. Clear glass plate at the end of 1 .

5. (r) and 5 (1) Indian ink figures. (Fixation points).

6. Camera-like box.

7. Black cardboard, closing 6.

8. (r) and 8 (1) Fluorescent figures. (Fixation points).

9. Blue glass plate.

10. Ground glass and opalescent glass plates.

11. Metal strip.

12. Compur shutter.

13. Frosted lamp.

14. Lamp housing.

15. Rod carrying the lamp.

16. Screw fixing the rod.

17. Regulator lamp.

18. Cover of the regulator lamp.

19. Diaphragm of the Compur shutter.

20. Lens inserted into the shutter.

21. Segments of the shutter. 